\title{
The effect of static scanning and mobility training on mobility in people with hemianopia after stroke: A randomized controlled trial comparing standardized versus non-standardized treatment protocols
}

\author{
Stacey George ${ }^{1 *}$, Allison Hayes ${ }^{2}$, Celia Chen ${ }^{3}$ and Maria Crotty ${ }^{1}$
}

\begin{abstract}
Background: Visual loss following stroke impacts significantly on activities of daily living and is an independent risk factor for becoming dependent. Routinely, allied health clinicians provide training for visual field loss, mainly with eye movement based therapy. The effectiveness of the compensatory approach to rehabilitation remains inconclusive largely due to difficulty in validating functional outcome with the varied type and dosage of therapy received by an individual patient. This study aims to determine which treatment is more effective, a standardized approach or individualized therapy in patients with homonymous hemianopia post stroke.
\end{abstract}

Methods/Design: This study is a double-blind randomized controlled, multicenter trial. A standardised scanning rehabilitation program (Neuro Vision Technology (NVT) program) of 7 weeks at 3 times per week, is compared to individualized therapy recommended by clinicians.

Discussion: The results of the trial will provide information that could potentially inform the allocation of resources in visual rehabilitation post stroke.

Trial Registration: Australia and New Zealand Clinical Trials Register (ANZCTR): ACTRN12610000494033

\section{Background}

Stroke is a leading cause of disability [1], and approximately $30-35 \%$ of people with stroke have a homonymous hemianopia [2]. Hemianopia is described as "blindness in one half the field of vision in one or both eyes" [3]. Literature suggests that natural recovery occurs in $50 \%$ of people, mostly in the first month with minimal recovery taking place after 6 months [2]. Visual loss, following a stroke can be temporary or permanent, and interfere with an individual's ability to perform daily living activities and live independently [4].

Visual training in homonymous hemianopia post stroke is recognized as an important part of rehabilitation [5].

\footnotetext{
* Correspondence: stacey.george@health.sa.gov.au

'Flinders University, Division of Rehabilitation, Aged Care and Allied Health, Repatriation General Hospital, Daw Park, South Australia, 5043, Australia Full list of author information is available at the end of the article

Fullist of author information is available at the end of the article
}

The project described in this manuscript is comparing the effects of two approaches to treating visual field loss in the subacute rehabilitation phase (week two - six months) following a stroke. Currently rehabilitation employs three key strategies including optical devices, compensatory training and visual restitution therapy [6]. The first strategy is to use optical devices, such as prism glasses, to displace the visual image. The efficacy of prisms has not been demonstrated due to the lacking of a standardized methodology and few controlled studies [7].

The second strategy is compensatory and aimed at enhancing eye movements and/or head movements. This takes a number of different forms including the training of oculomotor or saccadic movements, on a standardised computer screen, or devices such as the Dynavision [8], which incorporates a larger visual area, where head movements are required. Oculomotor scanning performance,

(C) 2011 George et al; licensee BioMed Central Ltd. This is an Open Access article distributed under the terms of the Creative Commons Attribution License (http://creativecommons.org/licenses/by/2.0), which permits unrestricted use, distribution, and reproduction in 
thus only requiring eye movement, has been linked to subjects reported visual difficulties in everyday life [9]. Training of oculomotor or saccadic movements has been demonstrated to lead to an improved visual search field and reading ability [10-12]. No large scale randomized controlled trials have been performed to date to validate this intervention [13]. Dynavision training has been demonstrated to lead to improvement in visual scanning in a single case study [14], and in a descriptive study of driving [15], but not in a small controlled study of driving in stroke [16].

The third strategy uses visual restitution therapy and aims to enhance neuroplasticity [17]. This method involves the stimulation of the 'borderzone' or 'transition zone' between the area of visual loss and the intact area of vision [18]. A systematic review found [13] inconclusive evidence to support visual restitution based therapy as a means of restoring visual fields following brain damage.

In Australia, most visual training employs compensatory eye and head movement based therapy but current delivery of vision rehabilitation is recommended by treating therapists and is thus subjective [19]. Therapies can vary from a comprehensive combined static and mobility training to the prescription of magnifiers to help with reading with minimal instruction of their use. Due to the wide variability in type and dosage of therapy received by an individual patient, the benefits of visual training in terms of compensation and improved performance in functional tasks are uncertain [20].

In response to the need for a structured therapy program of visual training in homonymous hemianopia post stroke, the Neuro Vision Technology (NVT) program has been developed which is a standardized therapy and involves both a static scanning training and a dynamic mobility component. The static scanning component uses the NVT device, (see figure 1) to assess for the

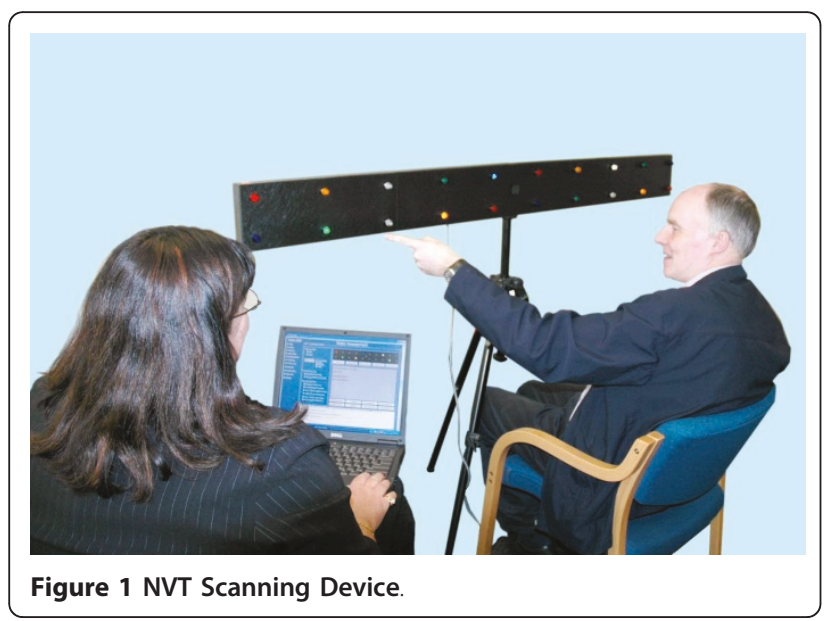

presence of neurological vision impairment, such as a homonymous hemianopia and/or visual spatial neglect. A standardized presentation of colored lights (LEDs) is displayed on a horizontal panel, placed at eye level approximately $30 \mathrm{cms}$ from the patient. The panel of lights extends approximately $80 \mathrm{cms}$ either side of central fixation, with the lights at the ends of the panel subtending an angle of approximately 70 degrees from midline. In the presence of a homonymous hemianopia the patient is required to use both head movement and eye movement towards the affected visual field to detect the light stimulus.

The NVT scanning device is used in rehabilitation to teach a pattern of systematic visual search strategies from the perimeter of the affected visual field towards the intact visual field. It aims to improve the patient's awareness and understanding of the nature of their vision impairment. The NVT training program then transfers visual scanning strategies, established using the NVT scanning device, into tasks that are mobility based. This developed NVT training differs from the other compensatory techniques as it involves a larger real visual world, whereas other compensatory training is aimed at rehabilitating reading abilities using a computer screen. NVT training has not been evaluated in terms of its effectiveness in rehabilitation for people with hemianopia.

To determine the most effective rehabilitation for people who have a hemianopia after stroke, it is necessary to examine the effects of the standardized and non-standardized rehabilitation strategies on performance in daily activities. We aim to compare the effectiveness of the standardized NVT program versus current individualized therapy as recommended by treating clinicians in patients with homonymous hemianopia post stroke.

\section{Methods and design}

The study design is a single-blind randomized, controlled trial in Adelaide, Australia, which will be conducted over 12 months. The intervention design is summarised in Figure 2.

\section{Randomized controlled trial Participants}

Patients will be included if they have had a stroke occurring a minimum of two weeks and a maximum of six months prior to the commencement of assessment; homonymous hemianopia (any field loss reached above and below the horizontal axis, as measured by Medmont C-100 perimetry [21] performed by a neurophthalmologist); score of 25 or more on the Mini Mental State Examination (MMSE) [22]; have corrected vision of at least 6/18; be able to mobilise a distance of 35 metres 


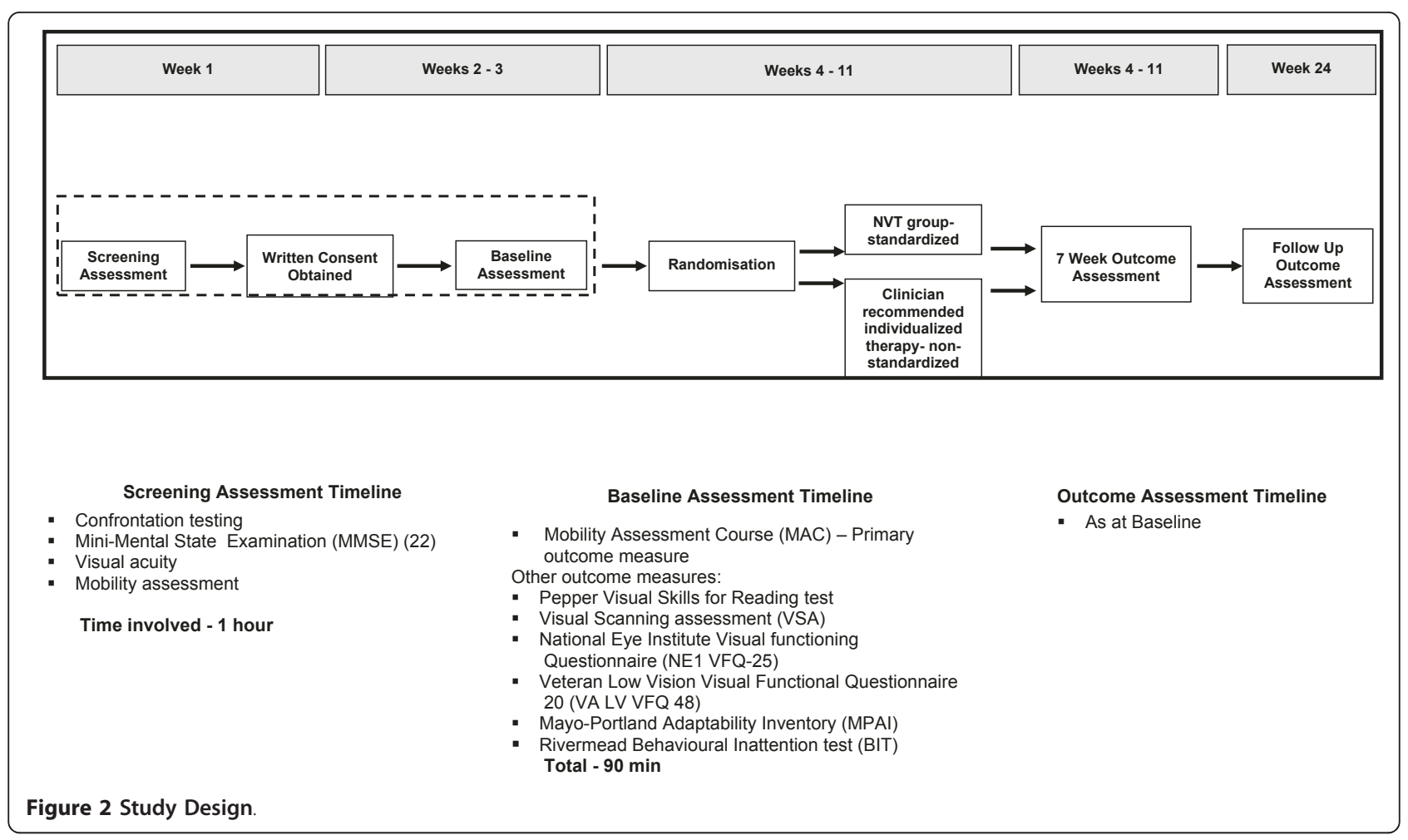

independently or with supervision or standby assistance; and be over 18 years of age. Formal assessment related to the inclusion criteria is detailed in Figure 2.

Patients will be excluded based on evidence of aphasia or poor English language skills that significantly impact on understanding instructions.

\section{Setting/Locations}

Participants will be recruited from acute hospitals, rehabilitation hospitals and outpatient settings in Adelaide, Australia.

\section{Procedures/Measurements}

All outcome assessments will take place face to face with participants by assessors who are blind to the allocation group. All outcome assessments (listed in Figure 2) will be performed by mobility instructors in vision rehabilitation who have received training in the standardized use of the tools.

\section{Recruitment}

Participants will be recruited from all three publicly funded rehabilitation facilities in Adelaide. In addition we will recruit from private rehabilitation facilities, neurophthalmologists and the two non government organisations who provide training for visual impairment in South Australia, Australia.

\section{Randomization}

Randomization is managed by the pharmacy department at the Repatriation General Hospital. A statistician external to the study will generate the random sequence using the random number generator in Microsoft Excel and created sequentially numbered, sealed envelopes containing group allocation for participants.

\section{Intervention 1}

Those participants who are randomized to the intervention group 1 will receive the standardized NVT program that consist of: three weeks static scanning training using the NVT scanning device and four weeks of mobility training using NVT scanning techniques.

All assessment and training tasks using the NVT scanning device are standardized using the Vision 2000 software program. Patient responses to the light stimulus are also recorded in a manner that allows for uniformity of performance measurement. The assessment process initially uses single light presentations to determine the patient's degree of spontaneous scanning into the affected field, and the degree of visual field loss. Subsequent light sequences utilize multiple lights in exercises, which are graded in complexity. Patient responses to these exercises are indicative of their ability to attend to multiple stimuli, their ability to adopt a systematic search pattern, the speed at which visual stimuli can be detected and the ability of the patient to utilize spatial reasoning to compare light patterns. The NVT assessment on the scanning device informs the NVT training process.

The NVT scanning device is used to teach a pattern of systematic visual search strategies from the perimeter of the affected visual field towards the intact visual field. 
These training exercises utilize patterns of lights that reinforce an efficient and functional speed of scanning to allow for accurate detection of all stimuli. Refer to Figure 1.

The NVT training program then transfers visual scanning strategies, established using the NVT scanning device, for example the amount of head turning and frequency, into the real world while walking, with the aim being for these strategies to become automatic. Scanning skills are graded from an environment that is structured and familiar to the patient, into more dynamic busy environments including shopping centres, where high level scanning skills and complex visual processing is required. Training will occur three times per week for 7 weeks. Audiovisual information of the intervention and NVT device is accessible through the following link: http://www.scivee.tv/node/16116.

\section{Intervention group 2}

Participants in intervention group 2 will receive individualized, or non-standardized therapy recommended by clinicians or usual care. "Usual care" for people receiving rehabilitation after stroke varies across inpatient and ambulatory rehabilitation units. However, intervention currently available includes one to one occupational therapy and mobility instruction that promotes visual scanning, activity of daily living skills, and mobility training from a non government organization, separate from health services, the Guide Dogs Association SA.NT. Techniques for intervention include pen and paper tasks, scanning training on light boards, compensatory training in activities of daily living and mobility. Dosage and type of therapy will be determined by the treating clinician and recorded. This group will be wait-listed for the NVT training which will be offered at the completion of the three month follow up.

\section{Outcomes}

As walking ability is a skill which is valued by those who have suffered a stroke and is required for safe discharge from hospital our primary outcome will be performance on the Mobility Assessment Course (MAC) [23] after three months. The MAC assesses performance on a mobility course with targets positioned on the walls along the course. Participants are scored on the number of targets they identify and the time taken to complete the course. The MAC, along with the Visual scanning analyzer (VSA) [23], were designed to test visual scanning. The MAC measures the extent to which a person visually scans and identifies hazards when walking, and has been validated with stroke patients [23]. In this validation study [23] the MAC included a measure of obstacle avoidance, which mobility evaluations generally include, however the results concluded that this was a poor measure of visual neglect in this population and thus was not used in MAC data collection in this study protocol.
The secondary outcome assessments will provide a broader picture of function and quality of life following a stroke and include Visual scanning analyzer (VSA) [23], Pepper Visual Skills for Reading test [24], National Eye Institute Visual functioning Questionnaire (NE1 VFQ-25) [25], Veteran Low Vision Visual Functional Questionnaire 20 (VA LV VFQ 48) [26], Mayo-Portland Adaptability Inventory (MPAI) [27] and the Rivermead Behavioural Inattention test (BIT) [28] as listed in Figure 2.

\section{Sample size}

Sample size has been calculated based on data reported in a pilot study validating the primary outcome measurement tool, the Mobility Assessment Course [23]. The minimum number of participants required is 20 , with 10 in each of the two groups providing $80 \%$ power (alpha level 0.05) to detect a mean difference 0.63 on scores, assuming the standard deviation is 0.74. Analysis of the results is planned in 2010 and will be undertaken by statisticians blind to the group allocation.

\section{Statistical analysis}

Data will be entered into an SPSS database with all identifying details removed. An ANCOVA will be performed to compare outcomes between the intervention groups, controlling for initial scores as covariates in the analyses.

Statistical analyses will be undertaken using SPSS version 17.0 Statistical software. All analyses will on an intention to treat basis and will be blinded (i.e. groups identified by number only) and results published according to the Consort Statement formal.

\section{Discussion}

Visual rehabilitation training is routinely provided for people with hemianopia following stroke however the benefits of visual rehabilitation training on functional outcomes have not been well evaluated. Despite us knowing it is important to rehabilitation as it impacts negatively on independence following stroke [4].

The goal of visual rehabilitation involves making the most of a person's residual vision, specifically to try to overcome the visual disabilities that are most troublesome to them. Part of the difficulty in evaluating the effectiveness of vision rehabilitation in homonymous hemianopia is the varied type of strategies used by therapists. At the moment the details of the rehabilitation program are negotiated between each patient and their rehabilitation therapist. No robust evidence currently exists to identify the most effective way to deliver vision rehabilitation in people with homonymous hemianopia after a stroke or the optimum time required [13].

There is evidence that providing more exercise therapy early after stroke improves peoples' ability to recover [29] but the effects of additional therapy in vision rehabilitation 
are unclear. No large scale randomized controlled trials have been performed to establish the effectiveness of visual rehabilitation following brain damage [13].

This project will compare the effectiveness of current visual rehabilitation protocols which vary in type and dosage according to individual clinicians' recommendations, to a new standardized technology (NVT). Functional outcome measures, such as performance on a mobility assessment course and scanning ability will be employed as well as patient reported outcomes such as, vision specific health related quality of life to allow a meaningful assessment of this rehabilitation intervention.

A debate exists around the mechanisms which underlie the remedial approach in visual rehabilitation following stroke, that is compensatory or neuroplasticity [7]. The outcome of our proposed study will contribute to the knowledge of the mechanisms behind the compensatory approaches, currently used in clinical practice. Furthermore, the results of the trial will provide information that will inform the allocation of resources in visual rehabilitation post stroke. This is in terms of dosage and type of therapy provided.

As in many areas of rehabilitation more rigorous research is needed to identify which remedial approaches should be included in clinical practice for visual loss following stroke.

\section{Acknowledgements}

Aspects of this study are funded by the Australian Governments National Eye Demonstration Grants Program, Foundation Daw Park, National Stroke Foundation and Flinders Collaborative Research Grant Scheme. Andrew Daly, from the Royal Society for the Blind has provided coordination for the project. Royal Society for the Blind mobility instructors and occupational therapists will provide the intervention and outcome assessment. Guide Dogs SA has provided control group intervention.

\section{Author details}

'Flinders University, Division of Rehabilitation, Aged Care and Allied Health Repatriation General Hospital, Daw Park, South Australia, 5043, Australia. ${ }^{2}$ Neuro Vision Technology, PO Box 141, Torrensville, South Australia, 5031, Australia. ${ }^{3}$ Department of Ophthalmology, Flinders Medical Centre and Flinders University, Flinders Drive, Bedford Park, South Australia, 5042 Australia.

\section{Authors' contributions}

All authors contributed to the design and conduct of the clinical trial. SG carried out data analysis, and drafted the manuscript. AH conceived of the study and participated in its design and coordination. $A D$ and $M C$ participated and oversees the trial process. CC is involved in the neuroophthalmic assessment and data acquisition of all participants and helped draft the manuscript. All authors read and approved the final manuscript. Written informed consent was obtained from the patient for publication of this case report and accompanying images. A copy of the written consent is available for review by the Editor-in-Chief of this journal.

\section{Competing interests}

Allison Hayes has participated in the development of the device and currently involved in the commercialisation.

Received: 20 April 2010 Accepted: 19 July 2011 Published: 19 July 2011
References

1. National Stroke Foundation: Facts and Figures [http://www.strokefoundation. com.au/facts-figures-and-stats], Accessed 11/9/08.

2. Zhang $X$, Kedar S, Lynn M, Newman N, Biousse B: Natural history of homonymous hemianopia. Neurology 2006, 66:901-905.

3. Dorland's Illustrated Medical Dictionary. Philadelphia, W.B. Saunders Company i, 23 1957, 599.

4. Denes G, Semanza C, Stoppa E, Lis A: Unilateral spatial neglect and recovery from hemiplegia. Brain 1982, 105:543-552.

5. Collopy D, Petherick M, Clarke G: A team approach to managing a patient with a neurological vision impairment. Journal of the Australasian Rehabilitation Nurses Association 2001, 4:16-20.

6. Roman JG: Progress in rehabilitation in hemianopic visual field defects. Cerebrovas Dis 2009, 27:187-190.

7. Pelak VS, Dubin M, Whitney E: Homonymous hemianopia: a critical analysis of optical devices, compensatory training, and novavision Current Treatment Options in Neurology 2007, 9:41-47.

8. Klavora P, Warren M, Leung M: Dynavision for rehabilitation of visual and motor deficits: A user's guide. Birmingham: visABILITIES Rehab Services Inc 1996.

9. Zihl J: Oculomotor scanning performance in subjects with homonymous visual field disorders. Visual Impairment Research 1999, 1:23-31.

10. Kerkhoff G, Munbinger U, Haaf E, Eberle-Strauss G, Stogerer E: Rehabilitation of homonymous scotomata in patients with postgeniculate damage of the visual system: saccadic compensation training. Restor Neurol Neurosci 1992, 4:245-254.

11. Pambakian ALM, Mannan SK, Hodgson TL, Kennard C: Saccadic visual search training: a treatment for patients with homonymous hemianopia. J Neurol Neurosurg Psychiatry 2004, 75:1443-1448.

12. Zihl J: Eye movement patterns in hemianopic dyslexia. Brain 1995, 118:891-912

13. Bouwmeester, Heutink J, Lucas C: The effect of visual training for patients with Visual field defects due to brain damage: a systematic review. J Neurol Neurosurg Psychiatry 2004, 75:1443-1448.

14. Klavora P, Gaskovski P, Heselgrave RJ, Quinn RP, Young M: Rehabilitation of visual skills using the Dynavision: A single case experimental study. Can J Occup Ther 1995, 62:37-43.

15. Klavora P, Gaskovski P, Martin K, Forsyth RD, Heselgrave RJ, Young M, Quinn RP: The effects of Dynavision on rehabilitation on behind-thewheel driving ability and selected psychomotor abilities of persons after stroke. Am J Occup Ther 1995, 49:534-542.

16. Crotty M, George S: Retraining visual processing skills to improve driving ability following stroke. Arch Phy Med Rehabil 2009, 90:2096-2102.

17. Kasten E, Sabel BA: Visual field enlargement after computer training in brain-damaged patients with homonymous deficits: an open pilot trial. Restor Neurol Neurosci 1995, 8:113-127.

18. McFadzean RM: NovaVision: vision restoration therapy. Curr Opin Opthalmol 2006, 17:498-503.

19. Teichman JC, Markowitz SN: Canadian research contributions to lowvision rehabilitation. Can J Ophthalmol 2008, 43(4):414-8.

20. Vasiliou C: Homonymous Hemianopia: Training compensatory strategies. Australian Orthoptic Journal 1990, 26:40-41.

21. Landers J, Sharma A, Goldberg I, Graham S: A comparison of perimetric results with the Medmont and Humphrey perimeters. Br J Ophthalmol 2003, 87:690-4

22. Folstein MF, Folstein SE, McHugh PR: Mini-Mental state: a practical method for grading the cognitive state of patients for the clinician. $J$ Psychiatr Res 1975, 12:189-198.

23. Verlander D, Hayes A, Mclnnes JK, Liddle RJ, Liddle GW, Clarke GERussell M, Ferguson W, Walsh PG: Assessment of clients with visual spatial disorders; a pilot study. Visual impairment research 2000, 2:129-142.

24. Stelmack J, Stelmack T, Fraim M, Warrington J: Clinical use of the Pepper Visual Skills for Reading Test in Low Vision Rehabilitation. American Journal of Optometry and Physiological Optics 2006, 64:829-831.

25. Stelmack JA, Stelmack TR, Massof RW: Measuring Low-Vision rehabilitation Outcomes with the NEI VFQ-25. Invest Ophthalmol Visual Science 2002, 43:9.

26. Stelmack JA, Szlyk JP, Stelmack TR, Demers-Turco P, Williams RT, Moran D, Massof RW: Measuring outcomes of vision rehabilitation with the Veterans Low Vision Visual Functioning Questionnaire. Invest Ophthalmol Visual Science 2006, 47:3253-61. 
27. Malec J: The Mayo-Portland Adaptability Inventory. The Center for Outcome Measurement in Brain Injury.[http://tbims.org/combi/mpai/], Accessed 27/2/09.

28. Wilson, Cockburn J, Halligan P: Development of a behavioural test of visuospatial neglect. Arch Phy Med Rehabil 1987, 68:98-102.

29. Kwakkel G, van Peppen R, Wagenaar RC, Wood Dauphinee S, Richards C, Ashburn A, Miller K, Lincoln N, Partridge C, Wellwood I, Langhorne P:

Effects of augmented exercise therapy time after stroke. A metaanalysis. Stroke 2004, 35:2529-36.

\section{Pre-publication history}

The pre-publication history for this paper can be accessed here: http://www.biomedcentral.com/1471-2377/11/87/prepub

doi:10.1186/1471-2377-11-87

Cite this article as: George et al:: The effect of static scanning and mobility training on mobility in people with hemianopia after stroke: A randomized controlled trial comparing standardized versus nonstandardized treatment protocols. BMC Neurology 2011 11:87.

\section{Submit your next manuscript to BioMed Central} and take full advantage of:

- Convenient online submission

- Thorough peer review

- No space constraints or color figure charges

- Immediate publication on acceptance

- Inclusion in PubMed, CAS, Scopus and Google Scholar

- Research which is freely available for redistribution

Submit your manuscript at www.biomedcentral.com/submit
C Biomed Central 